\title{
Pulmonary manifestations in HIV seropositivity and malnutrition in Zimbabwe
}

Michael O Ikeogu, Bart Wolf, Stanford Mathe

\begin{abstract}
Over a 10 month period 184 children, aged 5 years or less, who died at home had their nutritional status and HIV serostatus established; necropsies were also carried out. The HIV antibody test was positive in $122 / 184(66 \%)$. Of the HIV seropositive children Pneumocystis carinii pneumonia was present in $19(16 \%)$, cytomegalovirus pneumonia in nine $(7 \%)$, and lymphoid interstitial pneumonitis in $11(9 \%)$. Opportunistic infection was therefore seen in $28 / 122(23 \%)$ of the seropositive cases but in none of the seronegative cases. Tuberculosis was present in $8 / 184(4 \%): 6 / 122$ $(5 \%)$ in HIV seropositive and $2 / 62(3 \%)$ in seronegative children. Lung aspirate showed positive bacterial isolates in 106/ $122(86 \%)$ of HIV seropositive and $46 / 62$ (74\%) of seronegative children with Gram negative organisms predominating in both groups. Malnutrition was common and affected 106/184 (58\%); positive growth was obtained in $98(92 \%)$ of the malnourished children irrespective of their HIV serostatus. Malnutrition was significantly associated with bacterial lung infection after adjustment for the confounding effect of HIV status. No association was found between HIV serostatus and bacterial lung infection that could not be attributed to malnutrition at the time of death. The importance of adequate nutrition in reducing the risk of bacterial infection in HIV infected children is apparent. (Arch Dis Child 1997;76:124-128)
\end{abstract}

Keywords: pulmonary manifestations; HIV serostatus; malnutrition.

Pulmonary disease, particularly in the form of acute pneumonia, is a leading cause of death in children in developing countries where health care facilities are often inadequate and expert care not always easily accessible. With the present widespread infection with HIV the spectrum of pulmonary disease in children has become wider and accurate clinical diagnosis more difficult, often requiring complex laboratory facilities and invasive diagnostic procedures. Such facilities are not easily available in most developing countries. The problems have been compounded by the existence of widespread severe malnutrition due to socioeconomic deprivation in these countries, the symptoms of which often mimic HIV infection and both often coexist in the same patient, thereby resulting in a formidable diag- nostic and therapeutic challenge to the paediatrician.

Studies defining the various types of pulmonary disease in children with HIV infection are few and are mainly from industrialised countries, ${ }^{1-3}$ and those attempting to define the full spectrum are even fewer. ${ }^{4}$ Data obtained from industrialised countries may, however, not fully reflect the situation in developing countries. Although the vast majority of adults and children with HIV infection live in developing countries, there has been no comprehensive study of the spectrum of pulmonary disease in children in these countries and studies involving adult patients are few. ${ }^{56}$ The aim of the present study was to delineate the spectrum of pulmonary disease in HIV seropositive children from a developing country and to determine the differences, if any, from that in industrialised countries. It was also intended to study pulmonary findings in the presence of malnutrition in both HIV seropositive and seronegative children. The data presented here are part of a study on children dying at home and was approved by the Medical Research Council of Zimbabwe.

\section{Patients and methods}

The patients studied were children 5 years of age or less who were certified dead on arrival in hospital or who died shortly after in the casualty department of Mpilo Central Hospital, Bulawayo, at any time of the day or night. The survey covered a period of 10 months from July 1992 to April 1993. One of the authors (MOI or BW) was called immediately to see the child in the casualty department. Mpilo Central Hospital is a major referral and teaching hospital that serves a population predominantly from the lower socioeconomic groups.

A history was obtained from the parents or guardians to determine the events leading to the death of the child. The history included previous and current drug treatments. The body was examined as stipulated by law to exclude physical injury. Children dying from injury were excluded. Verbal consent was obtained from the parents or guardians on site after due explanation, and from the police department, to take specimens and to carry out a necropsy. Blood and lung aspirates were obtained in the casualty department before moving the body to the mortuary.

Aseptic technique was used to obtain all specimens of blood and lung aspirate. The whole trunk was cleaned with povidone-iodine solution and allowed to dry. Blood was taken from the heart by percutaneous puncture for 
Table 1 Demographic details of the children in the three groups; values are number (\%) except for age

\begin{tabular}{|c|c|c|c|}
\hline & Group $1(n=43)$ & Group $2(n=79)$ & Group $3(n=62)$ \\
\hline \multicolumn{4}{|l|}{ Gender } \\
\hline Male & $18 \quad(42)$ & $46 \quad(58)$ & $30 \quad(48)$ \\
\hline Female & $25 \quad(58)$ & 33 (42) & $32 \quad(52)$ \\
\hline Age (range) in months & $7.1(1-55)$ & $12.2(1-36)$ & $12.5(1-42)$ \\
\hline \multicolumn{4}{|l|}{ Nutritional status } \\
\hline Marasmus & $10 \quad(23)$ & $42 \quad(53)$ & $20 \quad(32)$ \\
\hline Underweight & $8 \quad(19)$ & 15 (19) & $11 \quad(18)$ \\
\hline Well nourished & $25 \quad(58)$ & $22 \quad(28)$ & $31 \quad(50)$ \\
\hline Histological evidence of pneumonia & $43(100)$ & $65 \quad(82)$ & 33 (53) \\
\hline
\end{tabular}

antibodies to HIV-1, which were determined by enzyme linked immunosorbent assay (ELISA; Vironostika, Organon, The Netherlands and Enzygnost, Behring, Germany). Lung aspirates were performed percutaneously as described by Hughes and Buescher ${ }^{7}$ from multiple sites (minimum of six, maximum of eight) to include all lobes using fresh disposable needles and syringes different from those used for taking blood. The same needle was used for puncturing all the sites without detaching it from the syringe. The lung aspirate was inoculated immediately directly on to chocolate and blood agar and incubated immediately at $37^{\circ} \mathrm{C}$. Bacterial identification in the first two days was by standard bacteriological method. Sterile plates were further incubated for 10 days. Only those children seen within three hours of death had lung aspirates performed. Neonates were excluded from the study.

Necropsies were carried out by one of us (SM) or members of his staff under his supervision. Specimens were taken from parts of the lung that appeared macroscopically abnormal for histological studies. The initial standard staining technique was with haematoxylineosin. Tissue with features suggesting Pneumocystis carinii were further stained with silver methenamine for confirmation. Cytomegalovirus was identified by the typical cytoplasmic or nuclear inclusion bodies. Special attention was paid to the identification of histological evidence of tuberculosis. Abnormal lung tissue without typical histological features of tuberculosis was further stained with Ziehl-Neelsen stain for the presence of acid fast bacilli. Tissue with typical features of tuberculosis were not further processed. In cases where the child was previously hospitalised and investigated for tuberculosis, the diagnosis of tuberculosis was made if gastric washing were found to contain acid fast bacilli on smear or culture. Lymphoid interstitial pneumonitis (LIP) was established by the presence of diffuse alveolar septal infiltrates by lymphocytes, plasma cells, and plasmacytoid lymphocytes sometimes with associated peribronchiolar infiltrates.

As HIV antibody test by ELISA was the only test used for determining HIV status it was not possible to distinguish with certainty between infected and uninfected children under 18 months of age as it is generally recognised that passively transfered maternal HIV antibodies could persist in the child up to this age. We therefore adopted a simple classification of the patients into the following categories: group 1
(43 patients): HIV seropositivity with associated opportunistic infection, disseminated tuberculosis or LIP, conditions often but not invariably associated with HIV infection; group 2 (79 patients): HIV seropositivity without associated conditions of group 1; and group 3 (62 patients): HIV seronegativity with or without other associated diseases. Malnutrition was defined in terms of the Wellcome classification. ${ }^{8}$

\section{STATISTICAL ANALYSIS}

Univariate analyses were performed in EpiInfo (World Health Organisation, version 5.0). Odds ratio with $95 \%$ confidence interval (CI) were used where appropriate and the MantelHaenszel $\chi^{2}$ test was used for stratified data. HIV status and nutritional status were considered alternately as exposure and confounding variables. Both were linked with HIV seropositivity and a higher risk of infections. Malnutrition is common in HIV infection and therefore a potential confounder for the association between HIV serostatus and bacterial lung infection.

\section{Results}

In the 10 month period of the survey there were 334 children 5 years of age or less who were certified dead in the casualty department. Of these children, $184(55 \%)$ had all the investigations and qualified for inclusion in the study. One hundred and fifty (45\%) of the deaths were excluded because the interval between the time of death and presentation exceeded three hours or the child did not have all the investigations carried out. This group did not differ significantly from the study population with respect to age, sex, body weight, or reported causes of death. There were $94(51 \%)$ boys and $90(49 \%)$ girls. The mean age for the study group as a whole was 11.1 months (range 1-55 months). The mean ages of HIV seropositive and seronegative children were 10.4 months (range 1-55 months) and 12.5 months (range 1-42 months) respectively. Demographic details of the children in the three groups are shown in table 1 . Histological evidence of pneumonia was seen in $108 / 122$ ( $89 \%)$ of HIV seropositive and $33 / 62(53 \%)$ of HIV seronegative patients (odds ratio 6.78; 95\% CI 3.02 to 15.38 ; $\mathrm{p}<0.001)$.

\section{GROUP 1}

The HIV antibody test was positive in $122 / 184$ $(66 \%)$ and negative in $62 / 184(34 \%)$ of the cases. Group 1 accounted for 43/184 (23\%) of the total number of children studied or $43 / 122$ (35\%) of all seropositive cases. Opportunistic infections were seen in $28 / 122(23 \%)$ of all HIV seropositive cases with $P$ carinii pneumonia accounting for $19 / 122(16 \%)$ and cytomegalovirus pneumonia $9 / 122(7 \%)$. Of the total of eight cases of tuberculosis, $6 / 122(5 \%)$ were seen in seropositive cases of which four $(3 \%)$ were disseminated. LIP was seen in $11 / 122$ (9\%) of the HIV seropositive cases. Opportunistic infections were seen predominantly among infants. Of the 19 infants with $P$ carinii pneumonia and the nine infants with cytome- 
Table 2 Bacterial isolates by lung aspirate in HIV seropositive and seronegative children

\begin{tabular}{|c|c|c|c|}
\hline Organisms & Group $1(n=43)$ & Group $2(n=79)$ & Group $3(n=62)$ \\
\hline \multicolumn{4}{|l|}{ Gram negative } \\
\hline Klebsiella spp & 6 & 22 & 16 \\
\hline Pseudomonas spp & 1 & 5 & 1 \\
\hline Coliforms & 1 & 7 & 7 \\
\hline Haemophilus influenzae & 1 & 1 & 3 \\
\hline Proteus spp & 1 & 1 & 1 \\
\hline Other enterobacteriaceae & - & 1 & 1 \\
\hline Mixed growth & 2 & 4 & 3 \\
\hline Subtotal (\%) & $12(28)$ & $41(52)$ & $31(50)$ \\
\hline \multicolumn{4}{|l|}{ Gram positive } \\
\hline Streptococcus pneumoniae & 6 & 4 & 4 \\
\hline Staphylococcus aureus & 2 & 6 & 4 \\
\hline Coagulase negative staphylococcus & 1 & 1 & 1 \\
\hline Group A streptococcus & 3 & - & 2 \\
\hline Group D streptococcus & - & 1 & 1 \\
\hline Streptococcus viridans & - & 2 & 1 \\
\hline Mixed growth & 1 & 1 & - \\
\hline Subtotal (\%) & $13(30)$ & $15(19)$ & $13(21)$ \\
\hline Mixed Gram positive and negative (\%) & $7(16)$ & $18(23)$ & $2(3)$ \\
\hline Total (\%) & $32(74)$ & $74(94)$ & $46(74)$ \\
\hline
\end{tabular}

galovirus pneumonia only one in each group was more than 6 months old. The cases with $P$ carinii had a mean age of 3.3 months (range 1-7 months) and the cases with cytomegalovirus had a mean age of 3.8 months (range 1-7 months). In the 11 cases with LIP the mean age was 12.7 months (range 3-55 months) and the four cases with disseminated tuberculosis had a mean age of 18.5 months (range 9-26 months). Bacteria were isolated in 15 of the 19 cases with $P$ carinii, in eight of the nine cases with cytomegalovirus pneumonia, and in seven of the 11 cases with LIP.

BACTERIAL ISOLATES AND HIV SEROSTATUS

Twenty one children had received or were receiving oral antibiotics, usually cotrimoxazole in the two weeks before death. Altogether a total of 188 organisms were isolated from the 184 patients. Bacteria were isolated from 106/122 (87\%) HIV seropositive (groups 1 and 2 ) and 46/62 (74\%) seronegative (group 3) children. Details of the various isolates are shown in table 2. Mixed bacterial growth, that is more than one organism isolated in any combination of Gram positive or Gram negative or a mixture of both was obtained in a total of 38 cases of which two cases had three organisms isolated. Altogether
79 mixed organisms were isolated. The commonest combination was Gram negative enterobacteriaceae with a Gram positive organism usually group A streptococcus, Streptococcus pneumoniae, or Staphylococcus aureus.

BACTERIAL ISOLATES AND NUTRITIONAL STATUS Malnutrition was common in the study population and $106(58 \%)$ of the 184 children were classified as malnourished. The nutritional status of the three groups is shown in table 1 . Bacteria were isolated from 98 (92\%) malnourished and $54(69 \%)$ well nourished children; the details are shown in table 3. A substantial number of children with opportunistic infection were well nourished: 16 out of 19 cases of those with $P$ carinii pneumonia and four out of nine of those with cytomegalovirus pneumonia. There was strong evidence for an association between malnutrition and bacterial lung infection at death after adjusting for HIV serostatus (Mantel-Haenszel odds ratio 5.19; $95 \% \mathrm{Cl} 2.17$ to $12.42 ; \mathrm{p}<0.001)$. No association was found between HIV serostatus and bacterial lung infection that could not be attributed to nutritional status at the time of death.

Table 3 Bacterial isolates by lung aspirates in relation to HIV serostatus and nutritional status

\begin{tabular}{|c|c|c|c|c|}
\hline \multirow[b]{2}{*}{ Organisms } & \multicolumn{2}{|l|}{ HIV seropositive } & \multicolumn{2}{|l|}{ HIV seronegative } \\
\hline & Malnourished $(n=75)$ & Well nourished $(n=47)$ & Malnourished $(n=31)$ & Well nourished $(n=31)$ \\
\hline \multicolumn{5}{|l|}{ Gram negative } \\
\hline Klebsiella spp & 20 & 8 & 10 & 6 \\
\hline Coliforms & 4 & 4 & 4 & 3 \\
\hline Proteus spp & 2 & - & 1 & - \\
\hline Other enterobacteriaceae & 1 & - & - & - \\
\hline \multicolumn{5}{|l|}{ Other Gram negative } \\
\hline Pseudomonas spp & 6 & - & 1 & - \\
\hline Haemophilus influenzae & 2 & - & 1 & 2 \\
\hline Mixed growth & 6 & - & 3 & - \\
\hline Subtotal (\%) & $41(55)$ & $12(25)$ & $20(65)$ & $11(35)$ \\
\hline \multicolumn{5}{|l|}{ Gram positive } \\
\hline Staphylococcus aureus & 3 & 5 & 2 & 2 \\
\hline Coagulase negative staphylococcus & 2 & - & 1 & - \\
\hline Streptococcus pneumoniae & 5 & 5 & 2 & 2 \\
\hline Streptococcus viridans & 1 & 1 & 1 & - \\
\hline Group A streptococcus & 2 & 1 & - & 2 \\
\hline Group D streptococcus & - & 1 & 1 & - \\
\hline Mixed growth & - & 2 & - & - \\
\hline Subtotal $(\%)$ & $13(17)$ & $15(32)$ & $7(22)$ & $6(19)$ \\
\hline Mixed Gram positive and negative (\%) & $16(21)$ & $9(19)$ & $1(3)$ & $1(3)$ \\
\hline Total $(\%)$ & $70(93)$ & $36(76)$ & $28(90)$ & $18(58)$ \\
\hline
\end{tabular}


TUBERCULOSIS

Tuberculosis was diagnosed in only eight cases at necropsy. Of these four had disseminated tuberculosis, and all were HIV seropositive; bacterial pneumonia with positive lung aspirate was present in two. Tuberculosis was localised to the lungs in four cases who were diagnosed with positive cultures from gastric washings that ranged from 6-117 colonies during previous hospitalisation. The cause of death in these four cases was bacterial bronchopneumonia. Two of these cases with isolated pulmonary tuberculosis were HIV seropositive. Therefore only two of the eight cases of tuberculosis were seronegative. All eight cases of tuberculosis were marasmic.

\section{Discussion}

The patients studied have similar clinical characteristics to hospitalised children in our unit where a large number present with acute pneumonia, which is the commonest cause of hospitalisation and death. Malnutrition is quite common and HIV seropositivity associated deaths quite prevalent, currently running at over $70 \%$ of the deaths in hospitalised children in our institution (unpublished personal observation). We therefore believe that the data obtained from this study will closely reflect that expected in our hospitalised child population. It is clear from the data presented that pulmonary disease in both HIV seropositive and malnourished patients is dominated by serious pneumonia caused by bacteria, $P$ carinii, cytomegalovirus, tuberculosis, and LIP.

Percutaneous lung puncture for bacterial culture remains the most certain way of identifying bacterial organisms responsible for pneumonia. The use of blood culture, however, as a means of detecting bacterial organisms associated with pneumonia has a low yield. Fagbule, in a series of 99 malnourished children in Nigeria, using lung puncture, isolated Klebsiella spp and Escherichia coli in $39.4 \%$ and $8.8 \%$ of the cases respectively and $S$ aureus in $30.3 \%$ of the patients. ${ }^{9}$ Silverman et al working also in Nigeria before the HIV era had similarly high yield with predominantly Gram positive organisms isolated using lung puncture ${ }^{10}$ while Nathoo et al in Harare, Zimbabwe, using blood culture only, recently recorded only $22 \%$ positive bacterial isolates in 443 hospitalised children, which included many HIV seropositive and severely malnourished children with pneumonia. ${ }^{11}$

The use of postmortem specimens for culture for bacterial isolation has its protagonists. ${ }^{12} 13$ Lung puncture for this purpose to our knowledge has not been used before in patients soon after death. The pattern and spectrum of isolates from our material is similar to that reported in live patients from lung puncture specimens and blood culture, thereby validating our technique and results. Gram negative organisms were isolated in a high proportion of cases with Klebsiella spp featuring most prominently in both HIV seropositive and malnourished children compared with well nourished children. Strict comparisons of results from different parts of the African con- tinent is not possible because of paucity of data. In the United States Gram positive organisms seem to dominate in some areas while Gram negative organisms feature more prominently in others. ${ }^{12414}$

$P$ carinii is an important cause of severe pneumonia in patients with acquired immunodeficiency, and children with HIV infection in industrialised countries are commonly infected with this organism..$^{1-3}$ Its role and incidence in developing countries has not been determined due to difficulties in diagnosis but our data suggest that it may be a relatively common infection in HIV seropositive infants. Nathoo et al studied a series of 704 children admitted to the University Teaching Hospital in Harare who had acute lower respiratory tract infection. ${ }^{11}$ Of these children, 219 had HIV infection diagnosed but the authors did not attempt to diagnose pneumonia due to $P$ carinii or any other opportunistic infection because of difficulties in clinical diagnosis, thereby illustrating the limitations of conventional clinical approach in the aetiological diagnosis of acute pneumonia in children.

$P$ carinii pneumonia has been diagnosed in immunocompetent infants mainly in the first months of life. ${ }^{15}$ With the use of antibody tests as the only means of determining HIV status, as was the case in our series, the presence of HIV antibody positivity and $P$ carinii pneumonia may not necessarily imply HIV infection and immunosuppression. The contribution of $P$ carinii to overall incidence of pneumonia and mortality in immunocompetent infants has not been established with certainty using modern accurate methods of diagnosis. However the fact that none of the children testing HIV seronegative in our series was found to have pneumonia due to $P$ carinii suggests that our cases with $P$ carinii pneumonia were most likely infected with HIV. In adult patients $P$ carinii pneumonia may he associated with bacterial pneumonia or tuberculosis. ${ }^{56}$ None of the children in our series had concomitant pulmonary tuberculosis but bacteria were frequently isolated.

Cytomegalovirus has the same clinical significance as $P$ carinii in that it is an opportunistic infection in the immunocompromised patient. Like $P$ carinii it has been described as a cause of pneumonia in immunocompetent infants. ${ }^{15}{ }^{16}$ In hospitalised patients it is difficult to differentiate clinically from $P$ carinii when the lung is the target organ. Our data suggest that cytomegalovirus pneumonia is not uncommon in HIV seropositive infants. Of therapeutic importance is the isolation of bacteria from the lungs in $P$ carinii and cytomegalovirus pneumonia as well as in cases with LIP. This has been documented also by others. ${ }^{124}$

Infection with Mycobacterium tuberculosis is common in developing countries and is usually pulmonary. Most data on tuberculosis and its association with HIV infection are from adult patients. ${ }^{5}{ }^{6}$ In one such survey from the Ivory Coast $^{5}$ it was the cause of death in $32 \%$ of adults with HIV infection and in $50 \%$ of similar patients in Harare. ${ }^{6}$ Accurate information on tuberculosis in children with HIV infection 
is scanty both in terms of prevalence as well as disease spectrum because of difficulties in diagnosis. Our study was the first from the African continent to attempt to establish the incidence of pulmonary tuberculosis in HIV seropositive children at necropsy. It must be noted that necropsy reports will not establish the actual incidence of a treatable condition such as tuberculosis in any population.

A recent report from Lusaka, Zambia, found 88 of $237(37 \%)$ of hospitalised children with various types of tuberculosis to be HIV seropositive compared with 26 of $242(10.7 \%)$ of children without tuberculosis. It is noteworthy that only 15 out $62(24 \%)$ of the seropositive children in Zambia who completed treatment showed improvement. ${ }^{17}$ In Abidjan, Ivory Coast, $289(6 \%)$ of 5180 consecutive cases of tuberculosis were found to be children under 15 years of age. The overall prevalence of HIV infection in these children was $11.8 \% .^{18}$ These two studies looked at the incidence of HIV seropositivity in patients with tuberculosis. There is need to establish the incidence of tuberculosis in HIV positive children. Our study had only a total of eight cases of tuberculosis of which only two were HIV seronegative. The rather lower than anticipated incidence of tuberculosis encountered in our series is not easily explained considering the worldwide concern about the resurgence of tuberculosis. This could be attributed to the type of the patient material used and the effect of high rate of immunisation of infants against tuberculosis in Zimbabwe. It is also evident from our study and those from Zambia and the Ivory Coast that the overall incidence of tuberculosis will vary in HIV positive patients depending on the type of patient population studied, the region, and most importantly the experience of the physician with the diagnosis of tuberculosis in children.

\section{Conclusion}

This series shows that opportunistic infection in HIV seropositive children is not uncommon in Africa. The main opportunistic infections are $P$ carinii and cytomegalovirus, which are frequently associated with bacterial pneumonia in our environment. These opportunistic infections were not seen in the absence of HIV seropositivity. Malnutrition was significantly associated with bacterial pneumonia irrespective of the HIV status of the child. No clear association was found between either HIV serostatus or bacterial pneumonia that could not be attributed to nutritional status in our study population, thereby emphasising the importance of adequate nutritional support. Surprisingly there were few cases of tuberculosis in these children at necropsy.

We are grateful to Dr E T Vos for statistical advice. Our sincere thanks to the superintendent of Mpilo Central Hospital, Dr N Chaibva, for permission to carry out the investigation and the nursing staff of the Casualty Department for their cooperation. nursing staff of the Casualty Department for their cooperation.
Many thanks to Mrs O Mdlongwa for her excellent secretarial assistance.

1 Rubenstein A, Morecki R, Silverman B, et al. Pulmonary disease in children with acquired immune deficiency syndrome and AIDS-related complex. $f$ Pediatr 1986;108:498-503.

2 Vernon DD, Holzman BH, Lewis P. Respiratory failure in children with acquired immunodeficiency syndrome and acquired immunodeficiency syndrome-related complex. Pediatrics 1988;82:223-8.

3 Glaser JH, Schuval S, Burstein O, Bye RB. Cytomegalovirus and pneumocystis carinii pneumonia in children with acquired immunodeficiency syndrome. F Pediatr 1992;120: 929-31.

4 Marolda J, Pace B, Bonforte RJ, et al. Pulmonary manifestations of HIV infection in children. Pediatr Pulmonol 1991;10:231-5.

5 Lucas SB, Hounnou A, Peacock C, et al. The mortality and pathology of HIV infection in a West African city. AIDS 1993;7:1569-79.

6 Pulmonary diseases in patients infected with human mmuno-deficiency virus in Zimbabwe, Central Africa. Trans R Soc Trop Med Hyg 1989;83:694-7.

7 Hughes WT, Buescher ES. Pediatric procedures. The respiratory tract. Philadelphia: WB Saunders, 1983:207-42.

8 Anonymous. Classification of infantile malnutrition. Lancet 1970;ii:302-3.

9 Fagbule DO. Bacterial pathogens in malnourished children with pneumonia. Trop Geogr Med 1993;45:294-6.

10 Silverman M, Stratton D, Diallo A, Egler LJ. Diagnosis of acute bacterial pneumonia in Nigerian children: value of needle aspiration of lung and of countercurrent immunoelectrophoresis. Arch Dis Child 1977;52:925-31.

11 Nathoo KJ, Nkrumah FK, Ndlovu D, et al. Acute lower respiratory tract infection in hospitalized children in Zimbapiratory tract infection in hospitalized chill

12 Eisenfeld L, Ermocilla R, Wirtschafter D, Cassady G. Systemic bacterial infections in neonatal deaths. Am $\mathcal{f} \mathrm{Dis}$ Systemic bacterial infecti

13 Klatersky J, Daneau D, Verhest A. Significance of routine postmortem bacteriological cultures. Pathol Biol (Paris) 1972;20:843-7.

14 Krasinski K, Borkowsky W, Bonk S, Lawrence R, Chandwani S. Bacterial infections in human immunodeficiency virus- infected children. Pediatr Infect Dis f 1988;7:23-8.

15 Brasfield DM, Stagno S, Whiteley RJ, et al. Infant pneumonitis associated with cytomegalovirus, chlamydia, pneumocystis and urea plasma. Follow-up. Pediatrics 1987;79:7683.

16 Smith SD, Cho CT, Brahmacupta N, Lenahan MF. Pulmonary involvement with cytomegalovirus infection in children. Arch Dis Child 1977;52:441-6.

17 Chintu C, Ganapati B, Luo C, Raviglione M, Diwan V. Seroprevalence of human immunodeficiency virus type 1 Seroprevalence of human immunodeficiency virus type 1 Infection is 7 1993;12:499-504.

18 Sassan-Morokro M, DeCock KM, Ackah A, et al. Tuberculosis and HIV infection in children in Abidjan, Cote d'Ivoire. Trans R Soc Trop Med Hyg 1994;88:178-81. 